\title{
Implantation of a Novel Cryopreserved Viable Osteochondral Allograft for Articular Cartilage Repair in the Knee
}

C. Thomas Vangsness Jr., MD ${ }^{1}$ Geoffrey Higgs, MD² James K. Hoffman, MS, MD, FAAOS 3 Jack Farr, MD ${ }^{4,5}$ Philip A. Davidson, MD $^{6}$ Farrell Milstein ${ }^{7}$ Sandra Geraghty, PhD $^{7}$

${ }^{1}$ Department of Orthopaedic Surgery, Keck School of Medicine, University of Southern California, Los Angeles, California

${ }^{2}$ Department of Orthopaedic Surgery and Sports Medicine, OrthoCare Institute, Lumin Health, Plano, Texas

${ }^{3}$ Department of Orthopaedic Surgery, Coordinated Health, Bethlehem, Pennsylvania

${ }^{4}$ Cartilage Restoration Center of Indiana, Ortholndy Hospital, Indianapolis, Indiana

${ }^{5}$ Department of Orthopedic Surgery, Indiana University School of Medicine, Indianapolis, Indiana

${ }^{6}$ Davidson Orthopedics, Salt Lake City, Utah

${ }^{7}$ Osiris Therapeutics, Inc., Columbia, Maryland

\author{
Address for correspondence C. Thomas Vangsness Jr., MD, \\ Department of Orthopaedic Surgery, University of Southern \\ California, Keck School of Medicine, 1520 San Pablo St, Suite 2000, Los \\ Angeles, CA 90033 (e-mail: vangsness@usc.edu).
}

J Knee Surg 2018;31:528-535.

\begin{abstract}
Keywords

- osteochondral allograft

- articular cartilage repair

- cryopreservation

- marrow stimulation

Restoration and repair of articular cartilage injuries remain a challenge for orthopaedic surgeons. The standard first-line treatment of articular cartilage lesions is marrow stimulation; however, this procedure can often result in the generation of fibrous repair cartilage rather than the biomechanically superior hyaline cartilage. Marrow stimulation is also often limited to smaller lesions, less than $2 \mathrm{~cm}^{2}$. Larger lesions may require implantation of a fresh osteochondal allograft, though a short shelf life, size-matched donor requirements, potential challenges of bone healing, limited availability, and the relatively high price limit the wide use of this therapeutic approach. We present a straightforward, single-stage surgical technique of a novel reparative and restorative approach for articular cartilage repair with the implantation of a cryopreserved viable osteochondral allograft (CVOCA). The CVOCA contains full-thickness articular cartilage and a thin layer of subchondral bone, and maintains the intact native cartilage architecture with viable chondrocytes, growth factors, and extracellular matrix proteins to promote articular cartilage repair. We report the results of a retrospective case series of three patients who presented with articular cartilage lesions more than $2 \mathrm{~cm}^{2}$ and were treated with the CVOCA using the presented surgical technique. Patients were followed up to 2 years after implantation of the CVOCA and all three patients had satisfactory outcomes without adverse events. Controlled randomized studies are suggested for evaluation of CVOCA efficacy, safety, and long-term outcomes.
\end{abstract}

received

May 25, 2016

accepted after revision

May 31, 2017

published online

July 24, 2017
DOI https://doi.org/

10.1055/s-0037-1604138. ISSN 1538-8506.
Copyright $\odot 2018$ by Thieme Medical Publishers, Inc., 333 Seventh Avenue, New York, NY 10001, USA Tel: +1(212) 584-4662.
License terms

(c) (1) $\ominus$ (\$) 
Affecting as many as 900,000 people annually in the United States, articular cartilage injuries remain a therapeutic challenge for orthopaedic surgeons. ${ }^{1,2}$ The most common treatment for articular cartilage damage is marrow stimulation. This reparative approach entails creation of channels to the subjacent marrow by abrading or perforating the subchondral bone. ${ }^{1}$ Marrow blood ascends up to the lesion bed, resulting in a blood clot containing mesenchymal stem cells (MSCs). Although it was originally believed that these MSCs would differentiate into chondrocytes and produce hyaline cartilage, more often fibrocartilage is formed., ${ }^{2,3}$ Fibrocartilage principally contains type I collagen rather than type II collagen (principle constituent of hyaline cartilage), resulting in poor long-term biomechanics. Although marrow stimulation may work well in small, shouldered lesions $\left(<2 \mathrm{~cm}^{2}\right)$, it often fails over time in larger lesions. ${ }^{1,2}$ Thus, although traditional marrow stimulation is easy to perform, minimally invasive, safe, and inexpensive, it has limited efficacy and often requires additional, invasive, and expensive revision surgeries.

Osteochondral allografts have long been successfully used as a restorative approach to treat articular cartilage damage. These articular cartilage and subchondral bone allografts are typically harvested as cylinders and can be combined to treat larger osteochondral lesions. The clinical use of fresh osteochondral allografts began more than 40 years ago and has shown good results. ${ }^{4,5}$ However, fresh stored osteochondral allografts must be used within 30 days due to rapid and significant decline of chondrocyte viability postharvest. ${ }^{6-9}$ In addition, osteochondral allograft implantation requires precise size and contour matching between donor and recipient tissues. The short shelf life, size-matched donor requirements, challenge of allogeneic bone healing, limited availability, and the relatively high price limit the use of fresh stored osteochondral allografts.

Here, we present a new option that combines reparative and restorative approaches for articular cartilage repair. The cryopreserved viable osteochondral allograft (CVOCA) (Cartiform, Osiris Therapeutics, Inc) contains full-thickness articular cartilage and a thin layer of subchondral bone $(<500 \mu \mathrm{m}$, estimated by histological analysis). The CVOCA maintains the intact native cartilage architecture with endogenous viable chondrocytes, chondrogenic growth factors, and extracellular matrix proteins to promote articular cartilage repair. ${ }^{10}$ Pores traverse the thickness of the osteochondral allograft, giving the CVOCA the versatility to be flexible to match the contour of various articular cartilage surfaces. The pores also enable the cryopreservation solution to better penetrate the thickness of the allograft, helping to preserve a high percentage of viable chondrocytes while in storage at $-80^{\circ} \mathrm{C}$ over its 2-year shelf life. ${ }^{10}$ The CVOCA builds upon more than 40 years of safety and efficacy of fresh osteochondral allografts as a restorative approach to articular cartilage repair. ${ }^{11-14}$ However, the CVOCA was designed as an adjunct to marrow stimulation. When the CVOCA is used with marrow stimulation, MSCs released from the patient's bone marrow are expected to initiate a reparative response within the pores of the CVOCA in the lesion in addition to the restorative approach from the allograft cartilage itself. ${ }^{10,15}$ The CVOCA may also be used with light abrasion to not weaken the subchondral bone, similar to a traditional osteochondral allograft but as an off-the-shelf product. The CVOCA is currently available in multiple sizes ranging from 0.8 to $4.5 \mathrm{~cm}^{2}$ with prices from $\$ 3,500$ to $\$ 11,000$. Using the straightforward, single-stage surgical procedure described here, the CVOCA may offer an important additional approach to articular cartilage restoration.

\section{Surgical Technique}

The lesion is defined by circumscribing the damaged cartilage slightly into the surrounding healthy tissue with a No. 11 or No. 15 blade, creating vertical lesion walls ( $\mathbf{- F i g . 1 A}$ ) in a manner as used for cell therapy (e.g., autologous chondrocyte implantation). A curette is used to debride any residual articular cartilage within the circumscribed boundary, completely removing the calcified layer and debriding back to a point where the cartilage-bone interface is stable (-Fig. 1B, C). The healthy articular cartilage surrounding the lesion should be moistened with normal saline often during the surgery. ${ }^{2}$

Next, the surgeon may choose to perform controlled marrow stimulation of the bony bed. To limit the damage to the subchondral bone, finer drill bits or wires are recommended.
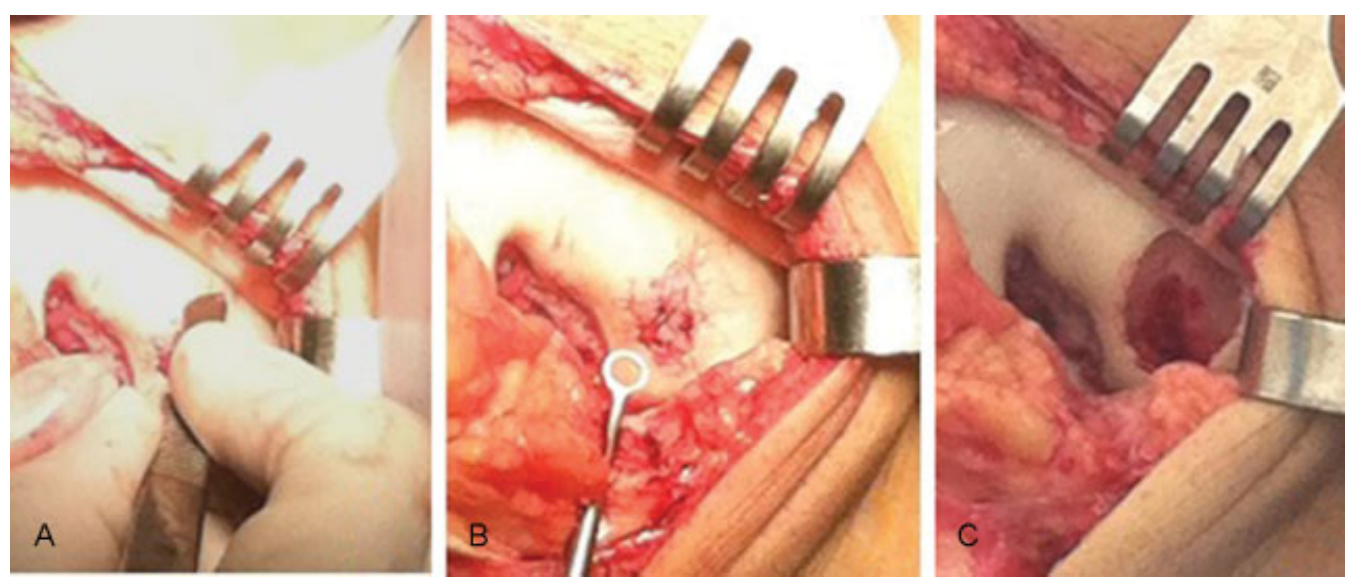

Fig. 1 (A-C) Intraoperative views of an articular cartilage lesion on the medial femoral condyle. The damaged cartilage is removed and the lesion bed is prepared for treatment. 

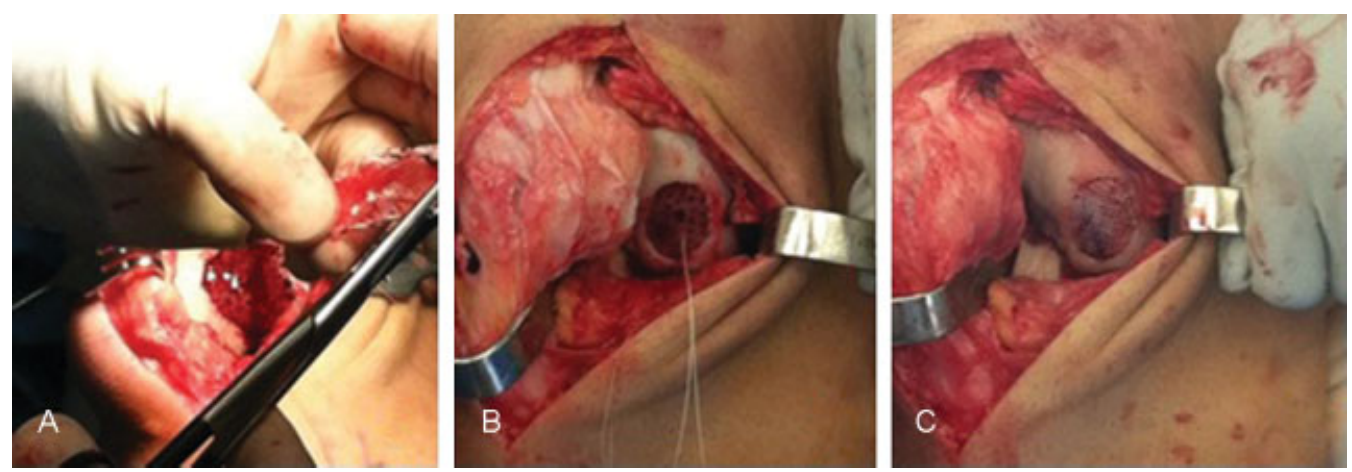

Fig. 2 Intraoperative views of the medial femoral condyle, demonstrating (A) sizing and (B, C) implanting the cryopreserved viable osteochondral allograft.

The marrow stimulation holes are placed at the perimeter of the lesion first, followed by additional holes inside at 2 to $3 \mathrm{~mm}$ intervals. For patella lesions, a small drill bit may be used due to the hardness of the subchondral bone. Alternatively, rather than creating holes, the base may be lightly abraded, removing only superficial bone from the subchondral bone plate to expose microscopic vascular arcades that create a "blush of blood" within this abraded region. Cooling irrigation is suggested to minimize thermal damage.

Once the lesion bed is prepared, a template of the lesion is made using either sterile foil or paper ( - Fig. 2A). Appropriate CVOCA sizes should be selected so that the CVOCA covers the entire lesion. Multiple sizes, including both circular and rounded rectangle shapes, provide options to fit a majority of lesions. For larger lesions, multiple CVOCAs can be trimmed and assembled on the template, placed side to side without stacking, to cover the entire defect. The CVOCA comes scored with a linear mark on the "deep" side to be approximated to the patient's bone to help the surgeon keep the graft oriented properly during the implantation. This orientation is key as the base will unlikely heal to the superficial cartilage zone if the CVOCA is inverted. The surgeon may mark the superficial side with a sterile marking pen for easy graft side recognition, ensuring that the pen is not chondrotoxic prior to use. The CVOCA is trimmed using a No. 15 blade or iris scissors to fit the custom template. The CVOCA is then trial fitted to the lesion to verify shape and size. The entire CVOCA should be in direct contact with the patient's underlying bone, closely apposed to the walls, and not proud to the surrounding cartilage. Nonmetallic and nonbone reactive suture anchors may be used to fix the CVOCA in place. Anchors which require a $3-\mathrm{mm}$ pilot hole or smaller should be chosen, and threaded with two 4-0 to 6-0 resorbable sutures ( $\mathbf{- F i g}$. 2B). The placement of the anchors is location dependent. A trochlea lesion requires placement of a central anchor to pull the graft down into place to take on the concave contour. Anchors around the periphery are beneficial for convex surfaces. The $4-0$ to $6-0$ resorbable sutures may also be used to secure the CVOCA to the surrounding cartilage. Damage to the native cartilage is prevented by following the curve of the needle. A thin, tapered, $17-\mathrm{mm}$, half circle needle is recommended.
Following placement of any suture anchors, the largest CVOCA is placed and secured, followed by any additional CVOCAs. The suture from each anchor is threaded through the holes in the CVOCA and tied tight enough to secure the CVOCA to the bone but not so tight as to cause damage (-Fig. 2C). The security of the graft construct is tested with palpation and by putting the joint through a range of motion (ROM) and observing movement of the graft. Fibrin glue is applied between the CVOCA periphery and the native cartilage walls, avoiding the holes in the graft, as a final step to secure the graft in place. Adherence to a rehabilitation protocol should be emphasized as failure to do so can lead to graft failure. In the case of immobilization, deep venous thrombosis prophylaxis measures should be taken.

\section{Patients and Methods}

Patients were considered for treatment if they presented with contained or uncontained focal articular cartilage lesions of the medial femoral condyle, the lateral femoral condyle, the trochlea, or the patella. Additional requirements for treatment included the presence of healthy surrounding articular cartilage with no degenerative articular cartilage disease, signs and symptoms consistent with the location of the chondral lesion, a lesion more than $2 \mathrm{~cm}^{2}$, and a physiologic age of younger than 60 years. Prior to surgical implantation of CVOCA, an arthroscopic assessment was performed on each patient to assess the articular cartilage lesion of interest and to evaluate the health of surrounding articular cartilage. Three patients with focal articular cartilage lesions were treated with the CVOCA. Magnetic resonance imaging (MRI) was evaluated by an external radiologist. Per regulations of the Department of Health and Human Services, a retrospective report containing three cases does not require Institutional Review Board approval. All HIPAA identifiers were removed from the data.

\section{Patient 1}

Patient 1 is a 52-year-old male ski instructor who presented with anterior cruciate ligament (ACL) deficiency, a large medial meniscus tear, and a $2.5 \mathrm{~cm} \times 1.5 \mathrm{~cm}\left(3.75 \mathrm{~cm}^{2}\right)$ contained grade IV osteochondral defect on the medial 

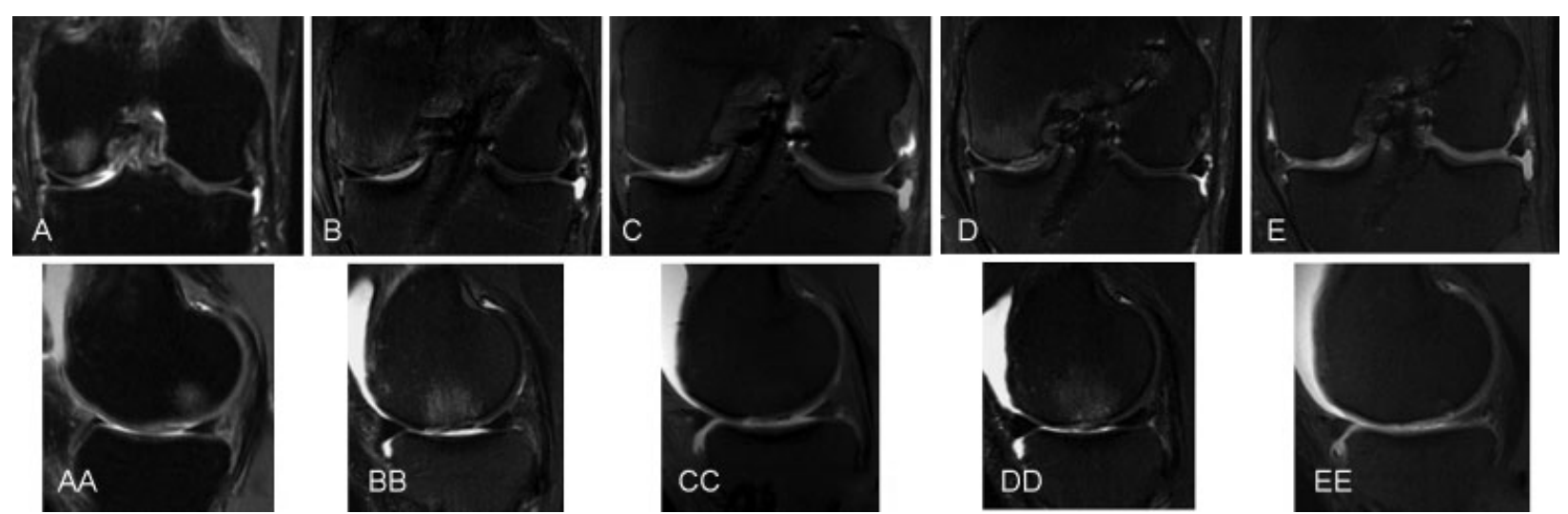

Fig. 3 Coronal and sagittal magnetic resonance images of patient 1 who was treated for a medial femoral condyle lesion, taken (A, AA) preoperatively, (B, BB) at 1 month postoperatively, (C, CC) at 3 months postoperatively, (D, DD) at 6 months postoperatively, and (E, EE) at 1 year postoperatively.

femoral condyle of the left knee. He underwent a superior compartment synovectomy, an ACL reconstruction using a bone-tendon-bone allograft, a repair of the medial meniscus, marrow stimulation of the medial femoral condyle, and an open CVOCA transplant to the medial femoral condyle of the left knee. MRI was performed on patient 1 preoperatively as well as at 1, 3, 6 months, and 1 year postoperatively.

\section{Patient 2}

Patient 2 is a 20-year-old male college student who presented with a $2.0 \mathrm{~cm} \times 2.5 \mathrm{~cm}\left(5.0 \mathrm{~cm}^{2}\right)$ osteochondral defect on the tibial plateau following a twisting injury of the left knee from skiing. He underwent an intra-articular synovectomy, debridement of his articular cartilage lesion, marrow stimulation of the lateral tibial plateau lesion, and arthroscopic-assisted CVOCA transplant to this area in the lateral tibial plateau of the left knee. The graft was held in place with peripheral fibrin glue. The lateral collateral ligament was taken down proximally for access as seen and repaired with a malleolar screw. MRI was performed on patient 2 preoperatively as well as at 1 month and 1 year postoperatively.

\section{Patient 3}

Patient 3 is a 28 -year-old female who presented with an osteochondral defect on the medial femoral condyle of the left knee following a traumatic bicycle injury. She underwent diagnostic arthroscopy with marrow stimulation of the medial femoral condyle, and an open CVOCA transplant to the contained lesion of the medial femoral condyle of the left knee. The lesion size was $1.5 \mathrm{~cm} \times 2.2 \mathrm{~cm}\left(3.3 \mathrm{~cm}^{2}\right)$. MRI was performed on patient 3 preoperatively as well as at 1 and 6 months postoperatively.

\section{Results}

\section{Patient 1}

Preoperative 3T MRI showed an osteochondral defect within the medial femoral condyle with bone marrow edema ( - Fig. 3A, AA). After the outpatient procedure, patient 1 underwent a routine postoperative course. He went to therapy at 3 weeks and began weight bearing at 8 weeks. The patient continued his progress with an increase in ROM and returned to activities of daily living, including employment and sports activity. He returned to work on crutches within 1 week and was driving comfortably at that time. As shown in - Fig. 3, a 1-month MRI showed that the CVOCA was in place with early evidence of resolution of subchondral edema. The CVOCA exhibited good surface congruity against adjacent cartilage, but the edges could still be visualized and areas of alteration in the underlying subchondral bone plate were still present. At 3 months, further integration of the CVOCA was evident by MRI. The CVOCA was in place and further reduction of bone marrow edema was observed. The 6-month follow-up MRI showed improved CVOCA integration with a few areas of altered signal characteristics in the CVOCA cartilage remaining when compared with the adjacent host cartilage (-Fig. 3D, DD). At 1 year, the signal within the CVOCA was more equivalent to adjacent host cartilage, the CVOCA appeared to completely fill the lesion with good incorporation and surface congruity, and the subchondral edema had resolved ( - Fig. 3E, EE). Comparison of the 1-, 3-month, and 1-year axial cut MRIs showed progressive incorporation of the CVOCA over time (-Fig. 4). The patient's ability to return to sports began at 6 months and increased all the way up to 1 year when he was skiing and surfing. He continued to progress and improve up to 2 years. His motion was complete and his pain was intermittent at most, with a pain-free outcome at 2 years.

\section{Patient 2}

The preoperative 3T MRI showed an osteochondral defect of the lateral tibial plateau with bone marrow edema ( - Fig. 5A, AA). Patient 2 was on crutches and returned to school the week following surgery. He went to physical therapy at school and he remained non-weightbearing for 8 weeks. In the 1-month follow-up MRI, the lesion was filled with good surface congruity, although the edges of the CVOCA were visible and the subchondral bone plate exhibited areas of alteration (-Fig. 5B, BB). The patient continued with his classes and progressed to sports 6 months after surgery. His progress continued to improve to a full ROM with minimal pain and 

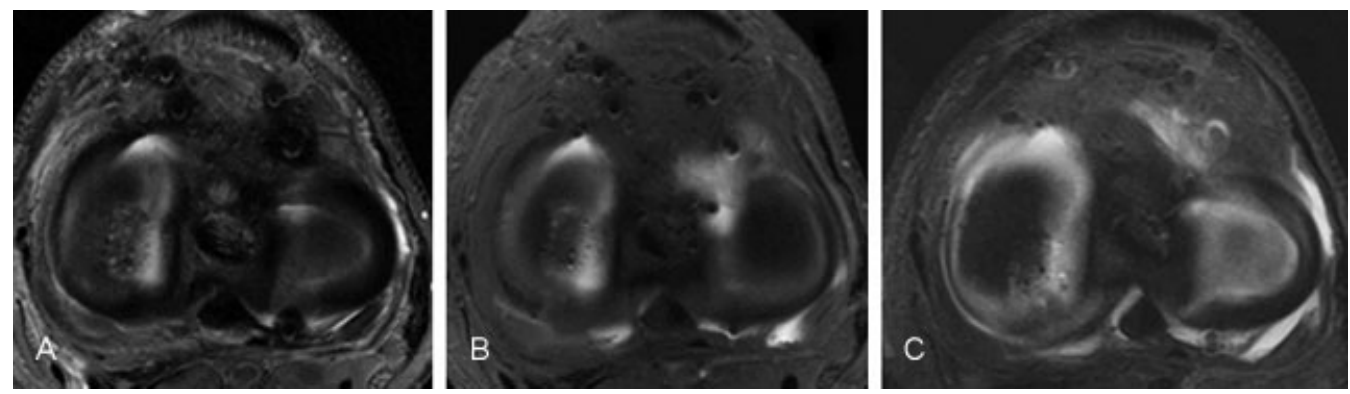

Fig. 4 Axial magnetic resonance images of patient 1 who was treated for a medial femoral condyle lesion, taken (A) at 1 month postoperatively, (B) at 3 months postoperatively, and (C) 1 year postoperatively.

his progress increased on the basketball court. The 1-year followup MRI showed complete fill with good surface congruity and CVOCA/host incorporation; only a couple small areas of altered signal characteristics were still evident within the CVOCA (-Fig. 5C, CC). Approximately 1 year after the surgery, the patient tore his contralateral ACL and went through reconstruction. Two years after surgery, his operated knee had minimal pain and complete unrestricted function.

\section{Patient 3}

The preoperative MRI showed an osteochondral defect about the medial femoral condyle with minimal bone marrow edema ( - Fig. 6A, AA). Patient 3 progressed after the outpatient surgery to return to work at 3 weeks in a non-weightbearing fashion. One month MRIs showed persistence of the CVOCA with near complete fill and good surface congruity, but increased fluid signal was noted in the underlying bone (-Fig. 6B, BB). The patient was walking at 8 weeks and she resumed her walking exercise patterns at 6 months after surgery. The 6-month MRIs showed improvements in the subchondral bone and continued integration of the CVOCA ( - Fig. 6C, CC). The patient continued to flourish at work with minimal complaints of pain when going up and down stairs and minimal clinical symptomology. She occasionally had effusions and pain at the end of long days of walking. Her WOMAC (Western Ontario and McMaster University Osteoarthritis Index) and IKDC (International Knee Documentation Committee) were above the 90th percentile at 2 years after surgery.

\section{Discussion}

A CVOCA was recently developed as a new treatment for articular cartilage lesions. The CVOCA is the only viable cartilage repair product on the market in the United States that can be stored for an extended period for immediate, point-of-care use. The CVOCA has been utilized to treat articular cartilage lesions since 2012. The only clinical peer-reviewed publications to date are a case report in the knee and a technique article for the ankle. ${ }^{15,16}$ Future controlled randomized clinical studies measuring functional
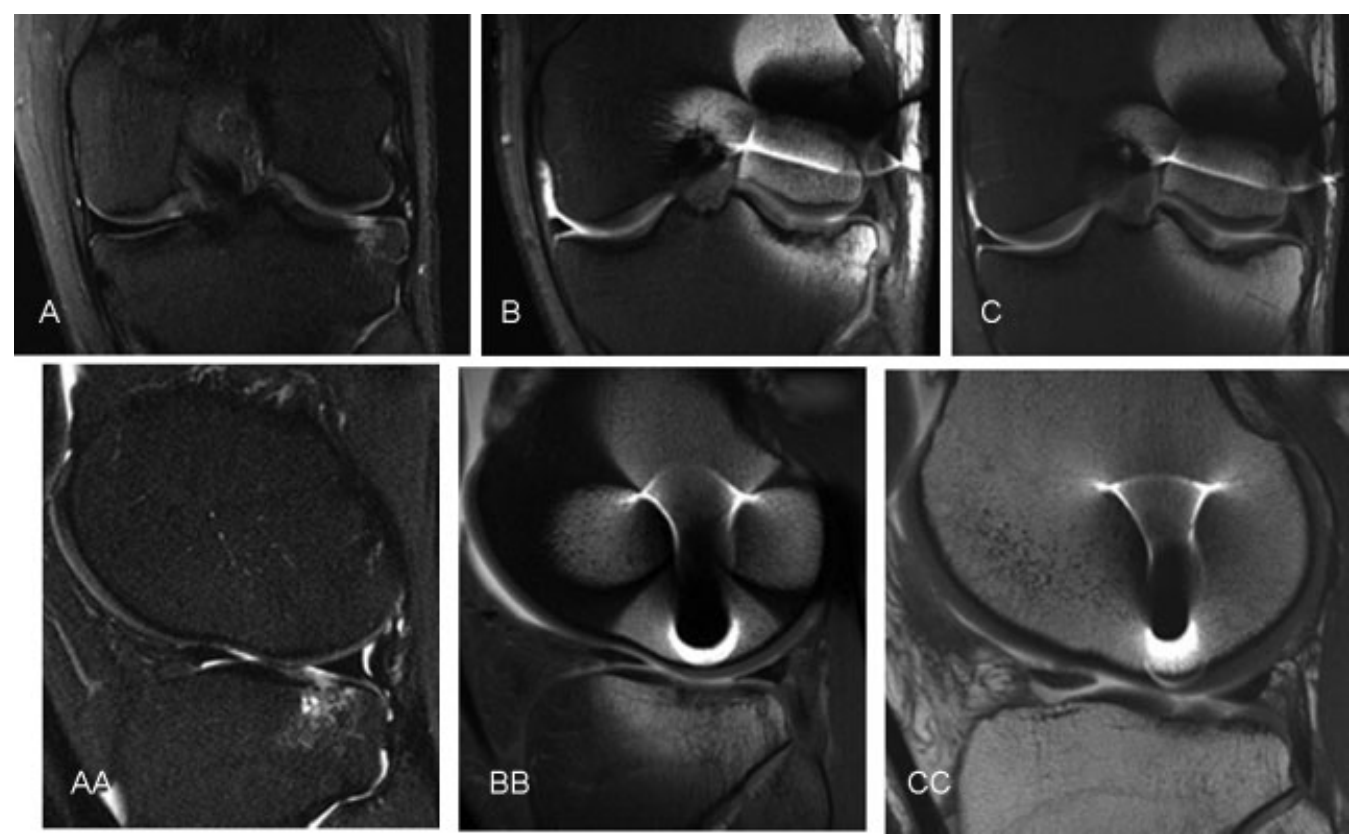

Fig. 5 Coronal and sagittal magnetic resonance images of patient 2 who was treated for a lateral tibial plateau lesion, taken (A, AA) preoperatively, (B, BB) at 1 month postoperatively, and (C, CC) at 1 year postoperatively. 

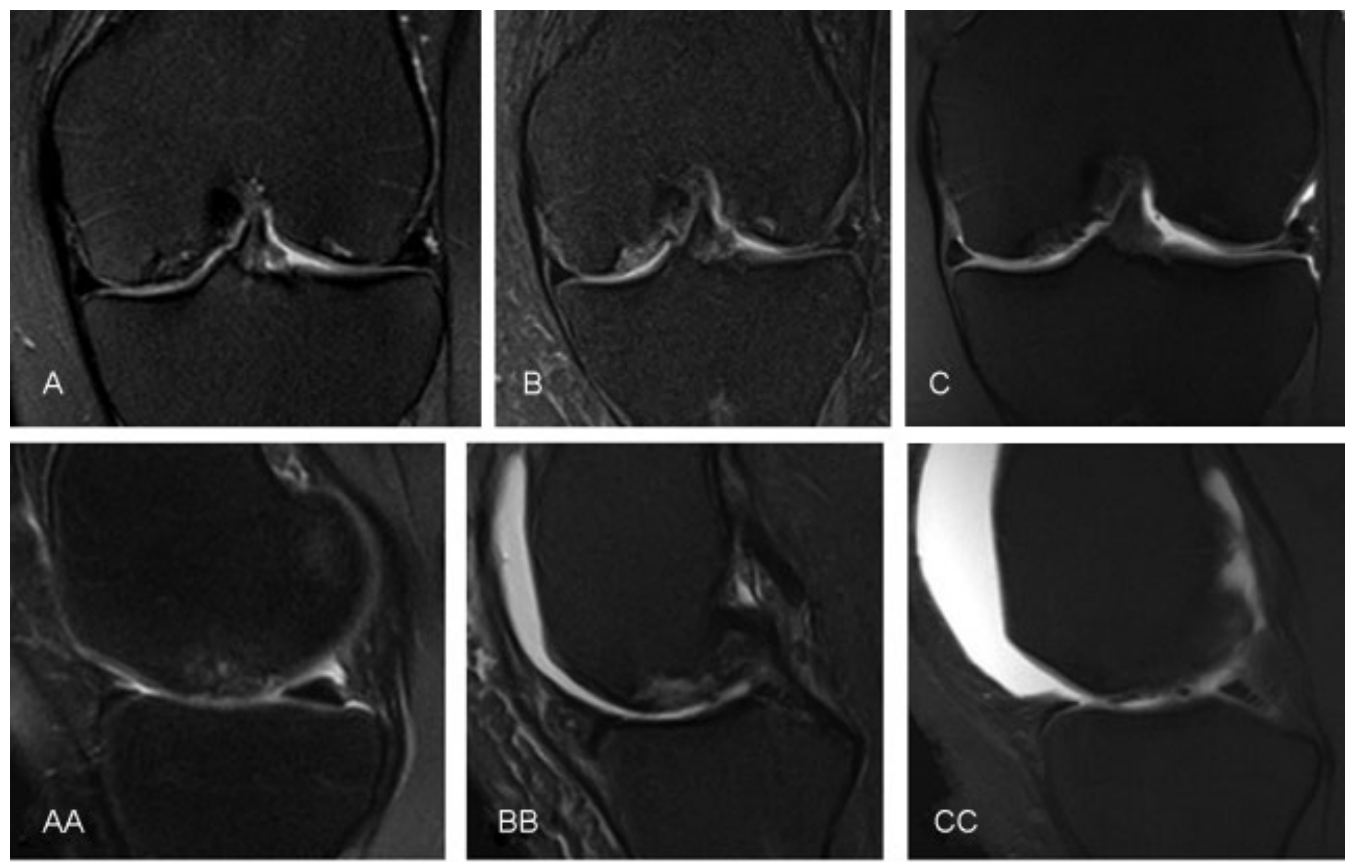

Fig. 6 Coronal and sagittal magnetic resonance images of patient 3 who was treated for a medial femoral condyle lesion, taken (A, AA) preoperatively, (B, BB) at 1 month postoperatively, and (C, CC) at 6 months postoperatively.

outcomes by validated methods are required to prove safety or efficacy of the CVOCA. The value of this study is a detailed description of the unique implantation technique for the CVOCA and outlining its application for repair of cartilage lesions at different sites in the knee through a summary of three clinical cases. The primary limitations of this case report are the small number of patients (three) with patient-reported outcomes and relatively short-term follow-up (2 years). However, these patients were some of the first to be treated with this new type of allograft and we believe the presentation of these initial outcomes provides valuable information to other surgeons considering using this new technique.

Although the surgical technique for marrow stimulation is very straightforward, the fibrocartilage that fills the lesion often breaks down over time leading to poor long-term outcomes, especially in older patients ( $>40$ years old) and in larger lesions $\left(>2 \mathrm{~cm}^{2}\right) \cdot{ }^{17-20}$ The surgical technique for the implantation of the CVOCA builds upon the surgical technique for marrow stimulation and can be performed without much added time or difficulty for an orthopaedic surgeon who is comfortable performing marrow stimulation alone. The CVOCA must simply be trimmed to fit the debrided lesion and secured in place with sutures and/or suture anchors. The addition of the CVOCA to a lesion treated with marrow stimulation has the potential to expand the lesion size able to be treated, as the CVOCA provides a microenvironment throughout the lesion that is favorable for MSC chondrogenesis and is expected to result in the formation of hyaline cartilage throughout the lesion rather than fibrocartilage. ${ }^{10}$ In this sudy, lesions ranging from 3.3 to $5.0 \mathrm{~cm}^{2}$ were treated with marrow stimulation augmented with the CVOCA. Multiple CVOCA units can be combined to treat lesions as large as $10 \mathrm{~cm}^{2}$. It is important to consider that the CVOCA adds to the cost of a marrow stimulation procedure, especially in cases involving large lesions where multiple grafts are required. The potential clinical benefits should therefore be considered carefully and weighed against the added procedural cost for each case. When lesions are properly selected, patients treated with marrow stimulation generally report symptomatic improvement over the first 2 years, similar to the improvements reported here. ${ }^{1}$ However, deterioration in functional outcomes is often evident starting at 18 months following marrow stimulation treatment. ${ }^{1}$ This decline is attributed to incomplete defect fill, poor integration of the repair tissue with the surrounding articular cartilage, and the inferior wear characteristics of the fibrocartilage repair tissue., ${ }^{1,20-22}$ In contrast, the patients treated with marrow stimulation augmented with the CVOCA exhibited near complete fill with good surface congruity against adjacent cartilage as early as 1 month following surgery according to MRI analysis. In another recently published case study, a trochlear lesion treated with marrow stimulation plus the CVOCA exhibited significantly more hyaline cartilage repair tissue (85\%) than a lesion treated with marrow stimulation alone (5\% hyaline cartilage) at 9 and 8 months, respectively, following surgery when biopsies were collected and analyzed. ${ }^{15}$ The patient treated with the CVOCA reported resolution of pain and returned to normal activity, whereas the patient treated with marrow stimulation alone required a revision surgery. ${ }^{15}$ In the only other published cases to date, Tan et al described improvements in pain scores for four patients treated with marrow stimulation augmented with the CVOCA for talus lesions. ${ }^{16}$

Traditional, fresh stored osteochondral allografts, which are often used to treat larger articular cartilage lesions, are used with several millimeters of subchondral bone attached, 
resulting in stiff grafts that must match the contour of the host cartilage surrounding the implant site. Harvesting and implanting fresh osteochondral allografts with the proper contour requires donor and recipient size matching, often taking time to acquire the properly sized graft, and specialized instrumentation and skill at the time of surgery. In contrast, the flexibility of the CVOCA enables the allograft to fit to multiple contours, allowing for immediate graft availability and a straightforward implantation procedure. Preservation using a cryoprotective agent such as glycerol or dimethyl sulfoxide has traditionally yielded only 20 to $30 \%$ chondrocyte viability in cartilage allografts with viable cells primarily located in the suprerficial zone. Clinical improvements have been shown to be proportional to viable chondrocyte content in transplanted osteochondral allografts. ${ }^{23-28}$ However, advances in the cryopreservation technique and a porated design, which optimized cryoprotectant penetration into the cartilage tissue, have resulted in high cell viability within the CVOCA (mean viability after thawing $=70.5 \%) .{ }^{10}$ Fresh osteochondral allografts are rarely implanted earlier than 14 days postprocurement due to donor screen requirements, and chondrocyte viability has been shown to decrease with storage time, reaching $\sim 70 \%$ viability by 28 days. $^{7,8,29,30}$ More recent studies have demonstrated high cell viability can be retained for up to 63 days following procurement by utilizing optimized storage conditions. ${ }^{31,32}$ The CVOCA is generally less expensive than a fresh osteochondral allograft when treating lesions $4.5 \mathrm{~cm}^{2}$ or smaller. The CVOCA ranges from $\$ 3,500$ for $0.8 \mathrm{~cm}^{2}$ of coverage to $\$ 11,000$ for $4.5 \mathrm{~cm}^{2}$ of coverage. In contrast, a fresh hemicondyle costs approximately $\$ 12,000$. A fresh osteochondral allograft is less expensive per square centimeter, making it more cost effective for large lesions. Long-term clinical outcomes and costs of the CVOCA in comparison to fresh osteochondral allografts remain to be evaluated.

The most similar treatment option to the CVOCA that is currently available in the United States is particulated juvenile allograft cartilage (DeNovo NT Graft, Zimmer Biomet), another off-the-shelf allograft cartilage product. In contrast to the CVOCA, this product is harvested from juvenile donors (vs. adult donors), minced into small pieces (vs. zonal structure intact), stored fresh in media at room temperature for up to 49 days after procurement (vs. cryopreserved within 4 days of procurement and stored for up to 2 years at $-80^{\circ} \mathrm{C}$ ), and implanted without marrow stimulation to augment the repair (vs. option to perform marrow stimulation). ${ }^{33}$ The cost per surface area of a treated defect for the two treatment options is similar. ${ }^{33,34}$ Although patient-reported outcomes following implantation of the particulated juvenile allograft cartilage are promising, the repair tissue often has a cobblestone-like appearance as the minced cartilage loses the biologically important zonal orientation of the superficial, transitional, and radial zones. ${ }^{33,35,36}$ Alternatively, structural maintenance within the CVOCA has been shown to translate to properly oriented repair tissue with distinct superficial, translational, and radial zones upon histological analysis in a preclinical animal model and a clinical biopsy. ${ }^{10,15}$ Larger studies with long-term outcomes are needed to better compare the two relatively new articular cartilage treatment options.

Although the outcomes highlighted in this report are early, all three patients treated with the CVOCA were satisfied with their resolution of symptoms. The surgical technique presented here for the knee has been applied to articular cartilage lesions in the talus ${ }^{16}$ and may be applicable to articular cartilage lesions in additional locations as well. Overall, the CVOCA builds upon the well-documented positives and negatives of two well-established techniques, marrow stimulation, and traditional osteochondral allografting, to offer surgeons a new treatment option for articular cartilage repair. Results presented in this study suggest that the CVOCA is safe for further long-term studies including a control/cohort and validated functional outcomes. Such studies are necessary to gain a better understanding of the clinical outcomes and pharmacoeconomical impacts of the CVOCA.

\section{Conflict of Interest}

None.

\section{References}

1 Mithoefer K, McAdams T, Williams RJ, Kreuz PC, Mandelbaum BR. Clinical efficacy of the microfracture technique for articular cartilage repair in the knee: an evidence-based systematic analysis. Am J Sports Med 2009;37(10):2053-2063

2 Farr J, Cole B, Dhawan A, Kercher J, Sherman S. Clinical cartilage restoration: evolution and overview. Clin Orthop Relat Res 2011; 469(10):2696-2705

3 Safran MR, Seiber K. The evidence for surgical repair of articular cartilage in the knee. J Am Acad Orthop Surg 2010;18(05): 259-266

4 Bedi A, Feeley BT, Williams RJ III. Management of articular cartilage defects of the knee. J Bone Joint Surg Am 2010;92(04): 994-1009

5 Görtz S, Bugbee WD. Allografts in articular cartilage repair. J Bone Joint Surg Am 2006;88(06):1374-1384

6 Langer F, Gross AE. Immunogenicity of allograft articular cartilage. J Bone Joint Surg Am 1974;56(02):297-304

7 Allen RT, Robertson CM, Pennock AT, et al. Analysis of stored osteochondral allografts at the time of surgical implantation. Am J Sports Med 2005;33(10):1479-1484

8 Pearsall AW IV, Tucker JA, Hester RB, Heitman RJ. Chondrocyte viability in refrigerated osteochondral allografts used for transplantation within the knee. Am J Sports Med 2004;32(01): 125-131

9 Williams RJ III, Dreese JC, Chen CT. Chondrocyte survival and material properties of hypothermically stored cartilage: an evaluation of tissue used for osteochondral allograft transplantation. Am J Sports Med 2004;32(01):132-139

10 Geraghty S, Kuang JQ, Yoo D, LeRoux-Williams M, Vangsness CT Jr Danilkovitch A. A novel, cryopreserved, viable osteochondral allograft designed to augment marrow stimulation for articular cartilage repair. J Orthop Surg 2015;10:66

11 Görtz S, Bugbee WD. Fresh osteochondral allografts: graft processing and clinical applications. J Knee Surg 2006;19(03):231-240

12 Demange M, Gomoll AH. The use of osteochondral allografts in the management of cartilage defects. Curr Rev Musculoskelet Med 2012;5(03):229-235 
13 McCulloch PC, Kang RW, Sobhy MH, Hayden JK, Cole BJ. Prospective evaluation of prolonged fresh osteochondral allograft transplantation of the femoral condyle: minimum 2-year follow-up. Am J Sports Med 2007;35(03):411-420

14 LaPrade RF, Botker J, Herzog M, Agel J. Refrigerated osteoarticular allografts to treat articular cartilage defects of the femoral condyles. A prospective outcomes study. J Bone Joint Surg Am 2009; 91(04):805-811

15 Hoffman JK, Geraghty S, Protzman NM. Articular cartilage repair using marrow stimulation augmented with a viable chondral allograft: 9-month postoperative histological evaluation. Case Rep Orthop 2015;2015(617365):617365

16 Tan EW, Guyton GP, Miller SD. Cartilage mesh augmentation technique for treatment of osteochondral lesions of the talus. Tech Foot Ankle Surg 2015;14(04):188-193

17 Xing L, Jiang Y, Gui J, et al. Microfracture combined with osteochondral paste implantation was more effective than microfracture alone for full-thickness cartilage repair. Knee Surg Sports Traumatol Arthrosc 2013;21(08):1770-1776

18 Steadman JR, Rodkey WG, Singleton SB, Briggs KK. Microfracture technique for full-thickness chondral defects: technique and clinical results. Oper Tech Orthop 1997;7(04):300-304

19 Mithoefer K, Williams RJ III, Warren RF, Wickiewicz TL, Marx RG. High-impact athletics after knee articular cartilage repair: a prospective evaluation of the microfracture technique. Am J Sports Med 2006;34(09):1413-1418

20 Kreuz PC, Erggelet C, Steinwachs MR, et al. Is microfracture of chondral defects in the knee associated with different results in patients aged 40 years or younger? Arthroscopy 2006;22(11): 1180-1186

21 Frisbie DD, Trotter GW, Powers BE, et al. Arthroscopic subchondral bone plate microfracture technique augments healing of large chondral defects in the radial carpal bone and medial femoral condyle of horses. Vet Surg 1999;28(04):242-255

22 Gudas R, Kalesinskas RJ, Kimtys V, et al. A prospective randomized clinical study of mosaic osteochondral autologous transplantation versus microfracture for the treatment of osteochondral defects in the knee joint in young athletes. Arthroscopy 2005; 21(09):1066-1075

23 Vangsness CT Jr, Garcia IA, Mills CR, Kainer MA, Roberts MR, Moore TM. Allograft transplantation in the knee: tissue regulation, procurement, processing, and sterilization. Am J Sports Med 2003;31(03):474-481
24 Abazari A, Jomha NM, Elliott JA, McGann LE. Cryopreservation of articular cartilage. Cryobiology 2013;66(03):201-209

25 Csönge L, Bravo D, Newman-Gage H, et al. Banking of osteochondral allografts, Part II. Preservation of chondrocyte viability during long-term storage. Cell Tissue Bank 2002;3(03):161-168

26 Ohlendorf C, Tomford WW, Mankin HJ. Chondrocyte survival in cryopreserved osteochondral articular cartilage. J Orthop Res 1996;14(03):413-416

27 Judas F, Rosa S, Teixeira L, Lopes C, Ferreira Mendes A. Chondrocyte viability in fresh and frozen large human osteochondral allografts: effect of cryoprotective agents. Transplant Proc 2007;39(08):2531-2534

28 Pallante AL, Görtz S, Chen AC, et al. Treatment of articular cartilage defects in the goat with frozen versus fresh osteochondral allografts: effects on cartilage stiffness, zonal composition, and structure at six months. J Bone Joint Surg Am 2012;94(21): 1984-1995

29 Malinin T, Temple HT, Buck BE. Transplantation of osteochondral allografts after cold storage. J Bone Joint Surg Am 2006;88(04): 762-770

30 Ball ST, Amiel D, Williams SK, et al. The effects of storage on fresh human osteochondral allografts. Clin Orthop Relat Res 2004; (418):246-252

31 Garrity JT, Stoker AM, Sims HJ, Cook JL. Improved osteochondral allograft preservation using serum-free media at body temperature. Am J Sports Med 2012;40(11):2542-2548

32 Stoker A, Garrity JT, Hung CT, Stannard JP, Cook J. Improved preservation of fresh osteochondral allografts for clinical use. J Knee Surg 2012;25(02):117-125

33 Tompkins M, Adkisson HD, Bonner KF. DeNovo NT allograft. Oper Tech Sports Med 2013;21:82-89

34 Tompkins M, Hamann JC, Diduch DR, et al. Preliminary results of a novel single-stage cartilage restoration technique: particulated juvenile articular cartilage allograft for chondral defects of the patella. Arthroscopy 2013;29(10):1661-1670

35 Bonner KF, Daner W, Yao JQ. 2-year postoperative evaluation of a patient with a symptomatic full-thickness patellar cartilage defect repaired with particulated juvenile cartilage tissue. J Knee Surg 2010;23(02):109-114

36 Farr J, Tabet SK, Margerrison E, Cole BJ. Clinical, radiographic, and histological outcomes after cartilage repair with particulated juvenile articular cartilage: a 2-year prospective study. Am J Sports Med 2014;42(06):1417-1425 